\title{
Experimenting clinical pathways in general practice: a focus group investigation with Italian general practitioners
}

\author{
Lucia Zannini, ${ }^{1}$ Cesarina Cattaneo, ${ }^{1}$ Paolo Peduzzi, ${ }^{1}$ Silvia Lopiccoli, ${ }^{2}$ Francesco Auxilia ${ }^{1,3}$ \\ ${ }^{1}$ Department of Biomedical Sciences for Health University of Milano; ${ }^{2}$ Health Management Unit \\ ASL Monza Brianza; ${ }^{3}$ Foundation IRCCS Ca' Granda Ospedale Maggiore Policlinico, Milano, Italy
}

\begin{abstract}
Significance for public health
Primary care is perceived as a crucial area in the delivery of health care because of the necessity to improve the sustainability particularly of public, universalistic health care systems moving from a hospital based resource consuming pattern of care. GPs are seen as a key part of a multidisciplinary team that can progressively assume a far more important role in the governance of their patients' pathways. The analysis of GPs attitudes and feelings about clinical pathways and clinical governance could help in making a step forward in this process; by considering suggestions and feelings expressed by professional bodies, policy makers can be helped in finding the best ways to meet their objectives in the medium term.
\end{abstract}

\section{Abstract}

Background. Clinical governance is considered crucial in primary care. Since 2005 , clinical pathways have been experimentally implemented at the Local Health Authority of Monza Brianza (ASLMB), Italy, to develop general practitioners' (GPs) care of patients affected by some chronic diseases. The experimentation was aimed at introducing clinical governance in primary care, increasing GPs' involvement in the care of their patients, and improving both patients' and professionals' satisfaction. In the period $2005-2006,12 \%$ of the 763 employed GPs in the ASLMB were involved in the experiment, while this percentage increased to $15-20 \%$ in 2007-2008.

Design and Methods. Twenty-four GPs were purposively sampled, randomly divided into two groups and asked to participate in focus groups (FGs) held in 2008, aimed at evaluating their perception of the experiment. The FGs were audio-recorded, dialogues were typed out and undergone to a thematic analysis, according to the Interpretative Phenomenological Approach.

Results. Four major themes emerged: i) clinical pathways can result in GPs working in a more efficient and effective fashion; ii) they can assure higher levels of both patient and professional satisfaction, since they sustain a caring approach and strengthen the GPs' role; iii) nevertheless, clinical pathways increase the bureaucratic workload and problems can arise in relationships among GPs and the LHA; iv) the implementation of clinical pathways can be improved, especially by reducing bureaucracy and by assuring their continuity.

Conclusions. Managerial aspects should be considered with care in order to experimentally introduce clinical pathways in general practice, and continuity of the experimentation should be guaranteed to improve GPs' adherence and commitment.

\section{Introduction}

Since the seminal work by Starfield, ${ }^{1}$ the need to confront the theme of remodelling health care systems, with attention to and investment in the development of primary care, has been repeatedly expressed. This requires a re-evaluation of the role of the hospital which should not be seen as the centre of the health system but rather as an integrated part of a network including both primary and secondary level of care. This alone can overcome the fragmentation of assistance that is so typical of complex health systems. ${ }^{2}$ Such a vision permeates national and regional decision-making. The recent Italian National Health Policy 2011-2013 gave the priority to the reorganisation of care procedures that focus on the general public and clinical governance. ${ }^{3}$

If correctly interpreted, clinical governance, as part of the reorganisation, must aim to provide an adequate response to health needs and a cohesive approach to shared professional guidelines. It is precisely in the context of primary care that clinical governance finds its greatest potential through taking responsibility not only for the single treatment, but for the entire course of assistance to patients with chronic diseases.

The development of guidelines to support the clinical management of chronic conditions, such as diabetes, has become a well-established practice. ${ }^{4}$ While guidelines are aimed at influencing the general practitioners' (GPs) behaviour in diagnostic and therapeutic decisions by widening physicians' knowledge, clinical pathways also promote the acquisition of skills (i.e. performing an ECG exam) in order to prevent, diagnose and cure some chronic diseases. They are both aimed at encouraging standardisation of good practices for all GPs. Despite this laudable aim, the implementation of guidelines/clinical pathways does not appear straightforward. ${ }^{4}$ Above all, just making the information available has shown itself to be insufficient to guarantee uptake of guidelines. ${ }^{5}$ In their systematic review, Majumdar et $a l^{6}{ }^{6}$ concluded that adoption of guidelines can be hindered by the very nature of evidence that is often uncertain, limited and complex. Furthermore, GPs can lack motivation to adopt guidelines because they are used to individually apply their own knowledge evidence and dealing with competing pharmaceutical incentives; this is in addition to consumers' demand or preference. Guidelines cannot be adopted due to lack of time, resources and incentives. For example, a research conducted with Australian GPs, who adopted guidelines in caring for diabetic patients, has suggested that incentives do promote better clinical management of those patients. ${ }^{7}$ Nevertheless, monetary reward is one influence, but so are practical considerations, the desire to do the right thing and having resources available. ${ }^{7}$ This means that, in order to promote clinical pathways, several supporting factors need to be in place. According to Saunders et al., these include: having a GP or other driver within a practice who is promoting clinical pathways, the avail- 
ability of a practice nurse, and access to computers and databases. Introducing clinical pathways in general practice requires changes to be made to practice systems. GPs often perceive these changes to be difficult to achieve in the midst of the pressures of patient workload. Lack of time and paperwork are often cited as major barriers to introducing new clinical pathways in general practice. Above all, a flexible approach to encouraging systematic management of chronic disease should be carried forward. Prescriptive incentives from the top down may have limited impact. ${ }^{7}$

As a consequence, interventions aimed at changing GPs' behaviour may not prove completely successful. ${ }^{8}$ Clinicians may prefer to manage patients in accordance with established routines ${ }^{9}$ that endorse their own autonomy. As Sanders et al. have stated, changing clinicians behaviour does not solely depend on the proven effectiveness of the new approach or its dissemination; contextual as well as occupational and professional factors, such as doctors' desire to retain their autonomy over clinical decision making, may be critical to any attempts at introducing innovations in health care. ${ }^{9}$

Some GPs correlate this autonomy to the quality of doctor-patient relationship: whilst the imposition of a rational basis for clinical autonomy might satisfy the requirements of the profession as a collective, it must struggle against a patient-centred rhetoric and a process of cognitive transformation that underpins everyday activity and the claimed autonomy of the individual clinician. ${ }^{10}$ From this point of view, observing guidelines may reduce the quality of the doctor-patient relationship.

Interestingly, in their research aimed at defining quality criteria in the management of common gastrointestinal disorders, starting from patients' views and practice guidelines, Jones et al. ${ }^{11}$ identified some key themes: better provision of information for patients, better communication with and access to secondary care providers, and structured follow up. These data seem to suggest that the introduction of guidelines in the management of common gastrointestinal disorders can improve the quality of the doctor-patient relationship.

In any case, locally agreed pathways may be followed in preference to national recommendations. In many countries, working groups composed of specialists, faculty members and GPs have been constituted in order to approve agreed pathways. For example, in Italy a working group on how to manage the hypertensive patient was set up. In 2009, it approved a clinical pathway on diagnostic tests, specialist evaluation, preventive and non-pharmacological treatments, and drug treatment of the hypertensive patient. ${ }^{12}$ Apart from being shared, guidelines are better accepted when they make the job easier. Accordingly, a qualitative study with English GPs in dealing with some guidelines, such as those published by the British Thoracic Society for the care of asthma, showed that they were welcomed. ${ }^{13}$ On the contrary, when guidelines result in an increase in the doctors' workloads, they are criticised. However, when English GPs found that the nurse could actually do the extra work required by a certain guideline, they greeted it with enthusiasm and agreed that it should have continued. ${ }^{13}$

Therefore, to improve guidelines, and consequently clinical governance, it is necessary to intervene on different levels, including the organisational model that characterises general practice. The examination of this model in the Italian context identifies the still limited use of associated forms in general medicine, while the experience of clinical governance, although promising, has not been sufficiently evaluated and validated. ${ }^{14}$ The experience at the Local Health Authority (LHA) of Monza Brianza (Azienda Sanitaria Locale di Monza Brianza, ASLMB) since 2005, aimed at introducing clinical pathways in general practice and is of interest for its size and complexity.

\section{Context of the study}

The clinical pathways' experimentation was carried out at the ASLMB since 2005. Here, 763 GPs were employed and over one million people were assisted. The experimentation was aimed to improve the care of patients with some chronic diseases through the definition of shared clinical pathways adopted at the different levels of care, according to a logical analysis of the system. The clinical pathways that were targeted in 2005 were chronic obstructive bronco-pneumopathy (COBP), diabetes, heart failure and patients undergoing anticoagulant therapy. Since 2007, clinical pathways for hypertension and depression have been added to the project. For all these pathologies, GPs were requested to provide diagnostic and/or therapeutic support to specialists' clinical practice.

Out of a total of 763 registered GPs, 95 (12\%) took part in the first phase of the experiment (2005-2006). Subsequently, (2007-2008) the number of GPs taking part in the project every year was between 15 and $20 \%$ of the total number of GPs employed in the ASLMB.

The objective of the project was to implement clinical governance of some chronic diseases through shared procedures of primary and specialised care, ${ }^{15}$ with an expected development of quality in primary care. The expected results of the experimentation were conforming GPs' practice to experimental clinical pathways and allowing them to become more involved in patients' primary care, improving compliance and patient satisfaction, as well as increasing GPs' professional satisfaction.

Specific use and adoption indicators (COBP, diabetes, heart failure, arterial hypertension, depression) were formulated to monitor and evaluate the pathways followed. Monitoring and evaluation showed an on average higher level of compliance to care standards and better results regarding patients' hospitalisation; this decreased in comparison with the hospitalisation of those patients not included in the clinical pathways experimentation.

For those patients who were included in the experimentation there was: i) a higher rate of diagnostic/therapeutic interventions, considered to be a means of tracking the clinical pathways; ii) an overall reduction in the use of use drugs and medicines and, therefore, lower expenditure per capita; iii) a reduction in specialised day hospital care; iv) fewer hospitalisations; and v) an improved compliance to prescribed treatments. This was also found in other contexts where clinical pathways have been implemented. ${ }^{16}$ The number of patients with chronic disease included in the project has increased from 2388 in 2005 (i.e. $4 \%$ of the total of ASLMB patients with the pathologies selected) to 16,889 in 2008 (11\% of the total). Those cared for within the project took advantage of a greater number of sessions, evaluated for the project as compliance tracking (e.g. in diabetes: electrocardiogram, fundus oculi testing, glycated hemoglobin, microalbuminuria). At the same time, there was a reduced rate of hospitalisation (both inpatient and day hospital). As far as patients' use of resources is concerned, data for 2005-2007 report that patients with diabetes, (COBP) and heart failure followed by doctors involved in the experimentation showed an overall trend for a decrease in the per capita expenditure for outpatient specialist care, hospitalisation and pharmaceutical assistance in the local area. $^{17}$

Besides objective indicators, it was considered useful to evaluate GPs' perception of the project. It is widely accepted that the opinion of those taking part in the experimentation is a fundamental component of its evaluation. 


\section{Design and Methods}

\section{Study design}

A qualitative study was designed to evaluate the GPs' perception of the clinical pathways' experience..$^{18}$ Qualitative research tends to focus on the meaning that a certain experience has had for certain people, exploring what factors influence making meaning of that experience. ${ }^{19}$

The study was aimed at encouraging GPs to reflect on their experience, at revealing critical issues and identifying possible lines of implementation. In addition, given the concept that adult education is mainly based on learning from experience ${ }^{20}$ and that this process is fostered by reflection, ${ }^{21}$ discussion with other GP colleagues was considered an important occasion for reflective learning on the clinical pathways' experience. Besides contributing in a practical manner to the evaluation of the project, the reflective practice fostered by focus groups has been set up as a strategy to develop GPs learning from their own personal experience and, therefore, an opportunity for continuing professional development.

\section{Participants}

A specific sampling procedure was carried out in line with the techniques of qualitative research sampling, that use non-representative sampling methods. ${ }^{18} \mathrm{~A}$ first group of participants considered to be important to the investigation identify other subjects who should be approached (snowballing sampling). Through this sampling technique, 24 physicians from ASLMB were recruited. These were considered expert informants about the clinical pathways' experience and were randomly subdivided into two Focus Groups (FG1 and FG2) which met in June 2008. After receiving the participants' informed consent and confirming confidentiality, the groups' discussions were recorded and transcribed verbatim. No patient data were collected. Ethical Committee approval was not sought, since in Italy this is not required for non-experimental studies involving healthcare professionals.

After the second FG, we noticed that the same ideas and feelings were reported about the clinical pathways experimentation by participants in both groups. Data saturation was, therefore, achieved with the two scheduled encounters and no more FGs were planned.

\section{Data collection}

Data were collected through focus groups. ${ }^{22,23} \mathrm{GPs}$ were invited by one of the investigators to participate in the study. All focus group meetings were led by two moderators who were supported by a third researcher, who took field notes.

The FG is a group discussion led by one or more interviewers following a specific questioning route. This is a data gathering technique used in qualitative studies, aimed at evaluating a particular experience, such as the introduction of guidelines in general practice. ${ }^{24-27}$ Besides recording their perceptions of the experience, the FGs provided opportunities for GPs to exchange their points of view. Hence, the FG was considered not only a means of collecting data, but also proved to be an approach that could encourage GPs' to reflect on their experience and, therefore, promote their professional development. For this reason, the two FGs were accredited as CME events.

The questioning route prepared for the FGs with the GPs taking part in the experimentation is shown in Table 1.

\section{Data analysis}

The complete transcriptions of the two FGs were analysed by two blinded researchers according to the Interpretative Phenomenological Approach. ${ }^{19}$ This method of analysis involves different steps:

the autonomous identification of some of the recurrent concepts in a text and their indexing (labelling);

the comparison between the two researchers of the identified labels, their homogeneity and their formalisation;

labelling all texts to be analysed according to the previously defined labels;

the autonomous grouping by each of the researchers of labels which are similar to each other in some categories;

the comparison between the researchers of the categories that emerged;

the autonomous identification by each of the researchers of possible macro-categories/themes which in turn include different categories; the comparison between the researchers of the emerged macro-categories to identify an agreement as to which categories come under which macro-category/theme.

Table 1. The questioning route of the focus group.

- Presentation of the chairmen and of aim and objectives of the focus group.

- Short introduction to the focus group

- Collection of the informed consents to taping of the meeting

- Information about policy on privacy

- Do you know each other? Would you introduce yourselves?

- How would you explain to a colleague GP from an other district the aim of this project? Let us try to express the meaning of it using a short sentence.

- What do you think in general about clinical pathways? Which relationship do you see between clinical pathways and the project you have got involved in?

- Which added value do you perceive for having been involved? What did you get?

- Instead which added value do you perceive for your patients? What did they get?

- Which choices, which activities could contribute to improve this project in your opinion? Please be as much precise as you can.

- How do you perceive the future development of this project?

- Let us summarize the main points that have emerged from the discussion...

- Do you want to make some other remarks?

- What is the final remark you want to say in order to make this project implemented by your district? 


\section{Rigour}

During FGs, the researchers continuously triangulated with participants their understandings of the gathered data.

Before analysing the transcripts, two researchers made explicit their pre-conceptions regarding the experience of clinical pathways. The researchers first performed all the steps of the data analysis individually and then discussed their findings together until an agreement was found. In each step of the analysis the researchers kept a reflexive attitude toward the phenomenon under study, considering continuously alternative interpretations of the participants' phrases and finally grounding their analysis on the transcriptions. ${ }^{28}$

\section{Findings}

Thirteen GPs took part in focus group 1 (FG1) and 11 GPs took part in focus group 2 (FG2). Average age of participants was 51.9 years in FG1 and 53.2 years in FG2. Median number of years of experience as a GP was 22 in FG1 and 23 in FG2. Average period of participation in the project of clinical pathways was 2.5 years for both FG1 and FG2. Therefore, both FG1 and FG2 are to be considered homogeneous for age, professional experience and participation in the project.

After analysing the two FGs by the method described above, a high consistency was found between labels, categories and themes that emerged from the two sessions. Results of the two FGs are, therefore, presented together while still indicating the interviewee code and the related FG that he/she attended. Four main themes emerged.

i) GPs perceive the clinical pathways in a positive light and see them as a means to acquire/make visible a rigorous, efficient and effective work method

GPs consider the clinical pathways' experience to be a positive one in so far as they represent an articulated project of clinical governance which, through the supply of diagnostic action and cure of chronic diseases, allows doctors to increase their involvement in caring for their patients and in their management:

The clinical pathway allowed us to concentrate on patient management within the outpatient unit and for the GP to become the case manager (GP1, FG2).

The clinical pathways' experience was considered to be positive for the doctors in so far as it provided a tool for their work, tied to the Evidence Based Medicine (EBM) guidelines; it made patient care and treatment more efficient and effective, and strengthened the GP's working method, while identifying skills which would otherwise remain hidden.

[The clinical pathways' experience] improved a methodology of work founded on a scientific basis and guidelines (GP6, FG1).

[It fosters] a scientific mentality (GP1, FG2).

[Clinical pathways' experiences] make the GPs' work more visible (GP1, FG2).

According to some interviewees, the clinical pathway experience was a positive one not only because it was able to improve the effectiveness of GPs' intervention, but also because it made their practice more efficient:

Patients' care was made more effective from a curative and therapeutic point of view while saving energy (GP9, GH1).

For these reasons, many GPs believe the clinical pathways experience represents a point of no return, mainly cultural (GP1, FG1), in that it designs:

The philosophy of the future (GP1, FG1) and provides

An essential model of caring for the patient to refer to (GP7, FG1).

ii) The clinical pathways' experience increased both patient's and doctor's satisfaction, since it sustains a caring approach and strengthens the GP's role
Many GPs agree that their participation in the project has allowed them to follow the patient more accurately (MMG6, FG1). This meant: Improved ability of taking care of the patient (GP5, FG1);

Patient satisfaction and appreciation (GP10, FG1);

A remarkable saving in terms of social costs of care for the patient (GP1, FG2).

In addition, the figure of the GP emerged clearly strengthened (GP4, FG1) because:

[Clinical pathways gave us] the possibility of widening knowledge and improving our method (GP6, FG1);

Improvement of our own performance and gain in credibility (GP3, FG2).

Furthermore, GPs established relationships with hospital specialists, acquired team work abilities and widened their professionalism.

A whole different world of relationships was opened up... We created a series of links [with specialists which created new] opportunities and possibilities which go beyond the clinical pathways' experience (GP3, FG2);

Specialists, colleagues and office personnel worked together (GP9, FG2).

Above all, the GPs felt they went back to filling a clinical role, which had been lost because of increasing bureaucracy in the Italian primary care system.

I had the sensation of having gained value as a doctor (GP7, FG2);

The good thing about the clinical pathways is taking back patient's management into our own hands, something that had been taken away from us (GP4, FG2).

iii) Clinical pathways' experiences increase the bureaucratic workload and problems can arise in relationships among GPs and LHA

Time and commitment required to the GPs for participating in clinical pathways' eperiences can be a heavy burden, since they require adherence to highly standardised procedures.

The clinical pathway created a standardised way to work with the patients (GP8, FG2);

The clinical pathway has clipped our wing" (GP9, FG2).

According to GPs, participating in the clinical pathways' experience involved a heavier bureaucratic workload, which could produce adverse effects:

[It was] a race against time (GP2, FG1);

I am very angry about the bureaucracy... Paper work... Forms to fill in (GP3, FG2).

Participating in the project did indeed require constant reporting to the LHA of the work carried out. Many GPs saw this as a hard task, since such reporting was so time consuming and was not sustained by adequate administrative and technological resources. The experimental project did, therefore, create some difficulties in GPs' relations with the LHA. These were mainly:

Difficulties in reporting and communications with the LHA personnel (GP12, FG1);

Difficulties in the LHA's monitoring of the situation (GP1, FG1).

iv) Implementation of the clinical pathways could be improved by slimming down the management administration procedures and guaranteeing continuity

To improve the implementation of the clinical pathways' experience in primary care, many interviewees mentioned the importance of management organisation. In particular, simplification and uniformity at all levels of organisation, promoting traceability and control of work undergone through reporting methods, which do not result in further management-administrative burden, were all considered crucial strategies to improve the implementation of clinical pathways in general practice. 
The LHA must provide a reporting method which facilitates the doctor's work (GP12, FG1);

It would be better to have clear indicators of results rather than having to keep piles of paper to be thrown away (GP3, FG2).

A further suggestion was to create an intermediary figure who operates between the GP and the LHA; somebody who is responsible for collecting the data relating to the experimental project. Furthermore, the LHA should better define the role of both the GP and the specialist, create a common project and a network, give space to the GPs, expanding their tasks in caring for the patients.

[We need] to define our role [...] and define the role of the specialists (GP1, FG1);

The leap in quality, in any case, can be made when the regional authorities or the Local Health Authority, or whoever, understands that they don't need to find additional resources for these experimental projects but they need to diversify the tasks (GP1, FG2).

In the midst of this process of improvement, a key point seems to be the need for continuity and the long-term profile of the project:

[It is important to have] the certainty that the project will continue (GP5, FG2).

Considering the efforts that are involved in the introduction of clinical pathways in general practice, it seems that GPs ask for guaranties in the continuity of the project, since they do not want their efforts to go to waste. According to GPs, the patients should also be more involved and must be educated to assume greater responsibility in the caring process. This could enable patients to make informed and shared choices related to the diagnostic or therapeutic decisions made by GPs participating in the experimentation.

If the citizen, who is at the centre of the system, is not educated helshe is not capable of participating in the choices about his/her health (GP3, FG2).

Furthermore, it is important to share the experience of the project among GPs and invest in training in order to involve a greater number of practitioners in the project.

[It is necessary to] hold specific courses, carried out seriously (GP10, FG2).

Finally, the interviewees pointed out the huge implications of the experiment regarding motivation. Sharing the experience among GPs, having the possibility of working together, receiving training on clinical pathways and, therefore, updating individual experience, are all elements which could make you feel less alone because, as one interviewee pointed out, working together, finding solutions together [for the problems that arise from clinical practice], are an excellent antidote to burn out (GP3, FG2).

\section{Discussion}

Since this is a qualitative study, no generalisations can be made of the findings, either for all the GPs who took part in this experimental project or for other GPs who are/will be involved in similar projects in the future. Nonetheless, the purposive sampling carried out (considered the gold standard for this type of study), together with the continuous debate and discussion with the participants of the emerging data during the FGs, make these results highly reliable. The study, therefore, does not give certainties, but rather identifies trends regarding the difficulties which may have to be met by administrators who intend to implement an experimental clinical pathway in contexts similar to the one described here. For this reason, this study can only provide some suggestions, to be verified in the field, to the design and implementation of experimental clinical pathways in other LHAs.

From the analysis of these findings, it emerges that the participants perceive the experiment to be a generally positive one. According to the participants, the experimental clinical pathways' experience not only results in a more effective and efficient treatment, restoring responsibility for the patient, but it also has a positive impact on the GP who, through this pathway, can apply scientific evidence of some of the diagnostic and therapeutic processes and adopt a more rigorous working method. This point seems to be related to the desire to do the right thing ${ }^{7}$ that has been shown to be a powerful motivational factor for Australian GPs in the uptake of guidelines. In fact, experimental clinical pathways' experiences, probably because of the evidence on which they are based and the work method which they foster, seem to allow a greater control of the work of each doctor, starting up processes of continuous improvement and self-training that can be expanded through audits. For these reasons, the Italian doctors who took part in this experiment consider the experimental clinical pathways to be a point of no return. The value of the methodology, acquired thanks to a clinical pathways' experience, could be an element to be put to good advantage when they are introduced and implemented in other LHA.

Clinical pathways can improve patient satisfaction with treatment and therapy. Italian doctors felt they regained their professional responsibility, that patients felt themselves to be followed more carefully, and believed that being able to benefit from diagnostic procedures in their GP's office makes those procedures easier to carry out and more comfortable. This result, in line with that of Jones et al., ${ }^{11}$ seems to suggest that the introduction of clinical pathways in general practice can improve doctor-patient relationships. Naturally, this is the GPs' point of view on patients' reactions to the clinical pathways' experimentation, and it should be examined in more detail with the patients, even though often doctors have reported their comments, directly using the same colloquial and dialectal language. Therefore, GPs suggest that an important purpose of the experiment, which was to heighten doctors' sense of responsibility in caring for their patients and increase their own satisfaction in the care delivered, has been reached. Furthermore, the experimental clinical pathways' experience seems to improve the GP's image, restoring his/her diagnostic/therapeutic profile, increasing professional satisfaction and creating the ideal conditions in which to improve their relationship with specialists. This means that also the purpose of putting professional satisfaction to good use seems to have been reached. These aspects of patients' and GPs' satisfaction should be taken into consideration in the phase of introduction/implementation of experimental clinical pathways.

Participation in the experiment showed, however, that there are some obstacles to its full realisation. Firstly, experimental clinical pathways require the creation of closer and more complex relationships both with colleagues, with specialists and with the LHA, which are not always easy to manage. Secondly, there are more important obstacles related to the extra amount of time required for the GPs to participate in the experiment, not only due to the extra diagnostic and therapeutic performed activities, but also due to the time spent in reporting those activities to the LHA (the so called paper work). This is in line with what has been pointed out by Saunders and colleagues. ${ }^{7}$ As a consequence, GPs claim they need greater resources to allow clinical management and that a lack of resrouces has a negative impact on their motivation, above all when scarsity of resources had to be managed alongside an increase in workload.

When introducing clinical pathways in general practice, it seems, therefore, important to pay particular attention to the aspects of administrative management, for example making reporting to LHA easier by slimming down procedures and training personnel and/or intermediary figures aimed at facilitating the reporting process. As far as organisation is concerned, GPs offer some indications of how to improve the experimental clinical pathways' experience. Firstly, by investing the money saved in hospital care in the general practice. One aspect which was particularly emphasised by the Italian GPs was the need to integrate teams of professionals and to give them adequate tools. GPs ask 
for a precise definition of their role and the work required of them by the experimental clinical pathway. Again, implementation of the clinical pathways in general practice seems to require the creation of a partnership between the different individuals involved. Patients' education and the development of a greater sense of clients' responsibility could represent a further investment that would put patients in a position to be able to exercise their own freedom of choice and, therefore, empowering them.

Finally, our experience seems to indicate the importance of the continuity of this type of experimentations; there is no room for improvisation. Once the experimental phase was concluded, the ASLMB has continued to propose similar projects year by year. Nonetheless, in spite of the positive results of the experience, the lack of guaranteed resources in the long-term to invest in the organisational development of primary care has created limitations as to how far the experience can be extended. This is in spite of the fact that many GPs have themselves asked for the clinical pathways' experience to be continued and extended.

\section{Conclusions}

Our FGs on the experimental clinical pathways' project in primary care carried out by the ASLMB reveal that on the whole the experience was perceived as a positive one. In fact, GPs declared that the experience had enhanced their caring attitude for the patients, had given them access to material and human resources that would otherwise have been inaccessible, and allowed them to work in direct contact with hospital specialists, with a consequent improvement in their own professional skills and image. Such benefits are perceived in contrast to the problems and obstacles concerning the highly standardised procedures that require additional temporal and economic resources for their management. These problems influence the GPs' motivation to adopt the guidelines proposed. In order to implement the experimental project, managerial aspects should be improved while at the same time its continuity should be guaranteed.

Finally, the focus group proved itself to be a valid tool that could be applied to data collection of the perceptions of the experimental clinical pathways' experience. It was also an effective strategy for comparison and discussion and, therefore, for GPs' continuing professional development.

Correspondence: Prof. Lucia Zannini, Department of Biomedical Sciences for Health University of Milano, via Pascal 36, 20133 Milano, Italy.

Tel. +39.02.50315101. E-mail: lucia.zannini@unimi.it

Key words: clinical governance, clinical pathways, continuing professional development, focus group, general practice

Acknowledgments: the Authors thank Dr. AP. Cantù and Dr D. Cereda who participated in the two focus groups as observers.

Contributions: LZ, conception and design; LZ, CC, FA, acquisition of data; LZ, CC data analysis and interpretation; LZ, CC, PP, SL, FA, article drafting and critical revision; FA, final approval of the version to be published.

Conflict of interests: the authors declare no conflict of interests.

Received for publication: 31 May 2012.

Accepted for publication: 31 July 2012.

(C) Copyright L. Zannini et al., 2012

Licensee PAGEPress, Italy

Journal of Public Health Research 2012; 1:e30

doi:10.4081/jphr.2012.e30

This work is licensed under a Creative Commons Attribution NonCommercial 3.0 License (CC BY-NC 3.0)

\section{References}

1. Starfield B, Shi L, Macinko J. Contribution of primary care to health systems and health. The Milbank Q 2005;83:457-502.

2. WHO. Primary health care now more than ever. 2008, WHO Publ., Geneva.

3. Ministero della Salute. Piano Sanitario Nazionale 2011-2013. Available from: http://www.salute.gov.it/pubblicazioni/ ppRisultati PSN.jsp

4. Kendall E, Sunderland N, Muenchberger H, Armstrong K. When guidelines need guidance: consideration and strategies for improving the adoption of chronic disease evidence by general practitcioners. J Eval Clin Pract 2009;15:1082-90.

5. Halbert R, Isonaka S. International primary care respiratory group (IPCRG) guidelines: integrating diagnostic guidelines for managing chronic respiratory diseases in primary care. Prim Care Respir J 2006;15:13-9.

6. Majumadar S, McAlister F, Furberg C. From knowledge to practice in chronic cardiovascular disease: a long and winding road. J Am Coll Cardiol 2004;43:1738-42.

7. Saunders M, Schattner P, Mathews M. Diabetes 'cycles of care' in general practice. Do government incentives help? Aust Fam Physician 2008;37:781-4.

8. Post EP, Kilboure AM, Bremer RW, et al. Organizational factors and depression managment in community-based primary care settings. Implement Sci 2009:4:84.

9. Sanders T, Foster NE, Ong BN. Perceptions of general practitioners towards the use of a new system for treating back pain: a qualitative interview study. BMC Med 2011;9:49.

10. Armstrong D. Clinical autonomy, individual and collective: the problem of changing doctors' behaviour. Soc Sci Med 2002;55:17717.

11. Jones R, Hunt C, Stevens R, et al. Management of common gastrointestinal disorders: quality criteria based on patients' views and practice guidelines. Br J Gen Pract 2009;59:415-21.

12. Donzelli A, Sghedoni D, Carelli F, et al. The clinical pathay for hypertensive patient of local health unit, hospitals and general practitioners, the Milan experience. Rev Recent Clin Trials 2011;6:16-23.

13. Checkland K. National Service Frameworks and UK general practitioners: street level bureaucrats at work? Sociol Health Ill 2004;26:951-75.

14. Auxilia F, Peduzzi P, Lopiccoli S, Vazzoler C. Percorsi di diagnosi e cura. Cure primarie e politiche sanitarie. Prospettive Sociali e Sanitarie 2011;9-10:6-10.

15. García MG, Mújica MP, Martínez Ocaña GC, et al. Results of a coordination and shared clinical information programme between primary care and nephrology. Nefrologia 2011;31:84-90.

16. Mitchell EA, Didsbury PB, Kruithof N, et al. A randomized controlled trial of an asthma clinical pathway for children in general practice. Acta Paediatr 2005;94:226-33.

17. Peduzzi P, Lopiccoli S. Governo clinico dei percorsi di diagnosi e cura. ASL 3, Regione Lombardia. 2008. Available from: http:/www.asImonzabrianza.it

18. Denzin NK, Lincoln Y. The SAGE Handbook of Qualitative Research. 2005, SAGE Publ., Thousand Oaks, USA.

19. Smith JA, Flowers P, Larkin M. Interpretative phenomenological analysis. Theory, Method and Research. 2009, SAGE Publ., London, UK.

20. Mezirow J. Transformative dimensions of adult learning. 1991, Jossey Bass Publ., San Francisco, USA.

21. Schön DA. Educating the reflective practitioner. 1991, Jossey Bass 
Publ., San Francisco, USA.

22. Corrao S. Il focus group. 2005, Franco Angeli Ed., Milano, Italy.

23. Zammuner V. I focus group. 2003, Franco Angeli Ed., Milano, Italy.

24. Cabana MD, Ebel BE, Cooper-Patrick L, et al. Barriers paediatricians face when using asthma practice guidelines. Arch Paediat Adol Med 2000;155:419-20.

25. Fairhurst K, Huby G. From trial data to practical knowledge: qualitative study of how general practitioners have accessed and used evidence about statin drugs in their management of hypercholesterolemia. BMJ 1998;317:1130.
26. Hutchinson A, McIntosh A, Cox S, Gilbert C. Towards efficient guidelines: how to monitor guideline use in primary care. Health Technol Asses 2003;7:18.

27. Wiener-0gilvie S, Huby G, Pinnock H, et al. Practice organizational characteristics can impact on compliance with the BTS/SIGN asthma guideline: Qualitative comparative case study in primary care. BMC Fam Pract 2008;9:32.

28. Mays N, Pope C. Qualitative research in health care. 1996, BMJ Publications, London, UK. 